\title{
Leptogenesis from tiny violation of Lepton Number
}

\section{Giorgio Arcadi*}

Max Planck Institut für Kernphysik, Saupfercheckweg 1, 69117 Heidelberg, Germany

E-mail: arcadi@mpi-hd.mpg.de

\section{Asmaa Abada}

Laboratoire de Physique Théorique, CNRS,

Univ. Paris-Sud, Université Paris-Saclay, 91405 Orsay, France

E-mail: abadadth.u-psud.fr

\section{Michele Lucente}

Centre for Cosmology, Particle Physics and Phenomenology (CP3)

Université catholique de Louvain, Chemin du Cyclotron 2, 1348 Louvain-la-Neuve, Belgium

E-mail: michele.lucente@uclouvain.be

The so-called ARS leptogenesis ensures a viable generation of the baryon asymmetry of the Universe by extending the Standard Model with GeV scale right-handed/sterile neutrinos which are being searched at present and next future experimental facilities. This kind of scenario is realized, in a technically natural way, in models, like the Linear and the Inverse see-saw, characterized by a tiny violation of the lepton number.

Neutrino Oscillation Workshop (NOW2018)

9 - 16 September, 2018

Rosa Marina (Ostuni, Brindisi, Italy)

\footnotetext{
*Speaker.
} 


\section{Introduction}

Some of the great puzzles of Modern Particle Physics, i.e. the Baryon Asymmetry (BAU) of the Universe, the Dark Matter (DM) component and origin of the very small masses of the Standard Model (SM) neutrinos, can be solved by extending the SM particle content with additional fermions, singlets under its gauge group, dubbed right-handed or sterile fermions.

Despite BAU via Leptogenesis can be achieved for a very broad range of masses of the new sterile fermions, it is tempting to focus on scenarios in which the mass of the new states is of the order or even below the Electroweak (EW) scale. This would be make them potentially testable at the LHC or at high intensity experiments like NA62 or the future planned DUNE and SHiP.

Right-handed/sterile neutrinos with masses not exceeding few tens of $\mathrm{GeV}$ can generate a viable BAU through the so-called ARS leptogenesis [1]. It consists into CP-violating oscillations, between the extra neutrinos, produced in the Early Universe through $2 \rightarrow 2$ and decay processes of SM states, but too weakly interacting to be into thermal equilibrium, and the SM (or "active") neutrinos. While these oscillations do not alter the total lepton number of the active+sterile system, asymmetries in individual active flavours are nevertheless created and subsequently converted into a baryon asymmetry by the Sphaleron processes. The minimal realization of the ARS mechanism requires the existence of a pair of highly degenerate sterile states with mass at the $\mathrm{GeV}$ scale. This apparently highly fine-tuned configuration is actually technically natural in some specific scenarios of the generation of the mass of SM neutrinos, the so-called Linear (LSS) and Inverse see-saw (ISS), where the small mass splitting of the two neutrinos is linked to a tiny violation of the lepton number. Notice that these mechanisms can naturally accommodate, in addition, a keV-scale mass DM candidate.

We will briefly show that within a combination of linear and inverse see-saw it is possible to achieve viable leptogenesis in a region of parameter space accessible to present and future experiments.

\section{Linear and Inverse See-Saw}

Two kind of models have been considered in our analysis. The first is a minimal model consisting into the extension of the SM with two right-handed neutrinos with opposite charges with respect to the lepton number, providing the following global neutrino mass term:

$$
-\mathscr{L}_{m_{v}}=n_{L}^{T} C \mathscr{M} n_{L}+\text { h.c., } n_{L} \equiv\left(v_{L}^{e}, v_{L}^{\mu}, v_{L}^{\tau}, N_{R}^{1 c}, N_{R}^{2 c}\right)^{T},
$$

where

$$
\mathscr{M}^{(v)}=\left(\begin{array}{ccc}
0 & Y v / \sqrt{2} & \varepsilon Y^{\prime} v / \sqrt{2} \\
Y v / \sqrt{2} & 0 & \Lambda \\
\varepsilon Y^{\prime} v / \sqrt{2} & \Lambda & \xi \Lambda
\end{array}\right),
$$

The terms in the mass matrix which violate the Lepton number are multiplied by factors $\varepsilon, \xi$, taken to be much smaller than 1 , with $Y, Y^{\prime}$ being the corresponding Yukawa couplings while $v$ is the Higgs vev. The mass matrix above arises from a combination of the LSS (Yukawa terms) and ISS (small Majorana mass) and it is then dubbed as LSS-ISS. The diagonalization of the latter mass matrix provides $m_{v} \simeq \varepsilon \frac{Y^{2} v^{2}}{\Lambda}$ for the light (SM) neutrinos. The remaining two eigenstates form a pseudo-dirac pair with mass $m_{\mathrm{PD}} \sim \Lambda$ and mass splitting $\Delta m_{\mathrm{PD}}^{2}=2 \xi \Lambda^{2}$. 
We have then considered pure realizations of the ISS. By this we intend a class of models, labelled as $\operatorname{ISS}\left(N_{R}, N_{S}\right)$, in which the SM is extended $N_{R}$ "right-handed" (i.e. with Yukawa interactions with the SM Higgs in the interaction basis) and $N_{S}$ "sterile" neutrinos (no interactions with the SM in the interaction basis). The mass spectrum of the theory, including the active neutrinos, is obtained by diagonalizing a mass matrix of the form:

$$
\mathscr{M}^{(v)} \equiv\left(\begin{array}{ccc}
0 & d & 0 \\
d^{T} & 0 & n \\
0 & n^{T} & \xi \Lambda
\end{array}\right)
$$

where $d, n, \xi \Lambda$ are complex mass matrices. The minimal phenomenologically viable realization of the ISS model is the ISS $(2,2)$ characterized, besides the active neutrinos, by two pairs of pseudodirac heavy states with mass splitting determined again by $\xi$. In addition, we have considered the ISS $(2,3)$ model which is characterized by the presence of an additional state with mass $m_{\mathrm{DM}}=\varepsilon \Lambda$ with very suppressed interactions with the SM states, to result cosmologically stable and then a potential DM candidate [2,3].

\section{Leptogenesis}

ARS leptongenesis can be described through a set of differential equations, in the density matrix formalism. Our numerical study is based on the equation defined in [4]: As detailed in [5], it is more convenient to use the variables $R_{N} \equiv \rho_{N} / \rho_{N, \text { eq }}{ }^{1}, \rho_{N}, \rho_{N \text {,eq }}$ being the density matrix for the heavy neutrinos and the one for thermal, and chemical potentials associated to $B-L$ :

$$
\begin{aligned}
& \frac{d R_{N}}{d t}=-i\left[\langle H\rangle, R_{N}\right]-\frac{1}{2}\left\langle\gamma^{(0)}\right\rangle\left\{F^{\dagger} F, R_{N}-I\right\}-\frac{1}{2}\left\langle\gamma^{(1 b)}\right\rangle\left\{F^{\dagger} \mu_{L} F, R_{N}\right\}+\left\langle\gamma^{(1 a)}\right\rangle F^{\dagger} \mu_{L} F, \\
& \frac{d \mu_{\Delta \alpha}}{d t}=-\frac{9 \zeta(3)}{2 N_{D} \pi^{2}}\left\{\left\langle\gamma^{(0)}\right\rangle\left(F R_{N} F^{\dagger}-F^{*} R_{\bar{N}} F^{T}\right)-2\left\langle\gamma^{(1 a)}\right\rangle \mu_{L} F F^{\dagger}+\left\langle\gamma^{(1 b)}\right\rangle \mu_{L}\left(F R_{N} F^{\dagger}+F^{*} R_{\bar{N}} F^{T}\right)\right\}_{\alpha \alpha},
\end{aligned}
$$

where $\left\langle\gamma^{i}\right\rangle$ are thermally averaged rates defined in [5] while $F_{\alpha I}=Y_{\alpha i} U_{i I}, U$ being the matrix which diagonalizes the neutrino mass matrix. The chemical potentials $\mu_{L}=\mu_{e, \mu, \tau}$ are related to the ones associated to $B-L$ by $\mu_{L_{\alpha}}=A_{\alpha \beta} \mu_{\Delta \beta}$, $A$ being a constant matrix given in [5]. The baryon comoving abundance $Y_{B}$ can be expressed as function of $\mu_{\triangle B}$ as:

$$
Y_{B}=\frac{28}{79} Y_{B-L}, \quad Y_{B-L}=\frac{45 N_{D}}{12 \pi^{2} g_{s}} \operatorname{Tr} \mu_{\Delta}
$$

The system of equations 3.1 has been solved for a large set of model points obtaining by performing scans over the parameter space of the LSS-ISS, ISS(2,2) and ISS $(2,3)$ models and imposing compatibility with neutrino oscillation parameters as well as from current laboratory searches of heavy neutrinos.

\footnotetext{
${ }^{1}$ Leptogenesis occurs at much higher temperatures with respect to the Majorana mass term. It is possible then to distinguish heavy neutrino-antineutrino states $N, \bar{N}$. System 3.1 features also an equation for $R_{\bar{N}}$ which has been omitted for simplicity.
} 

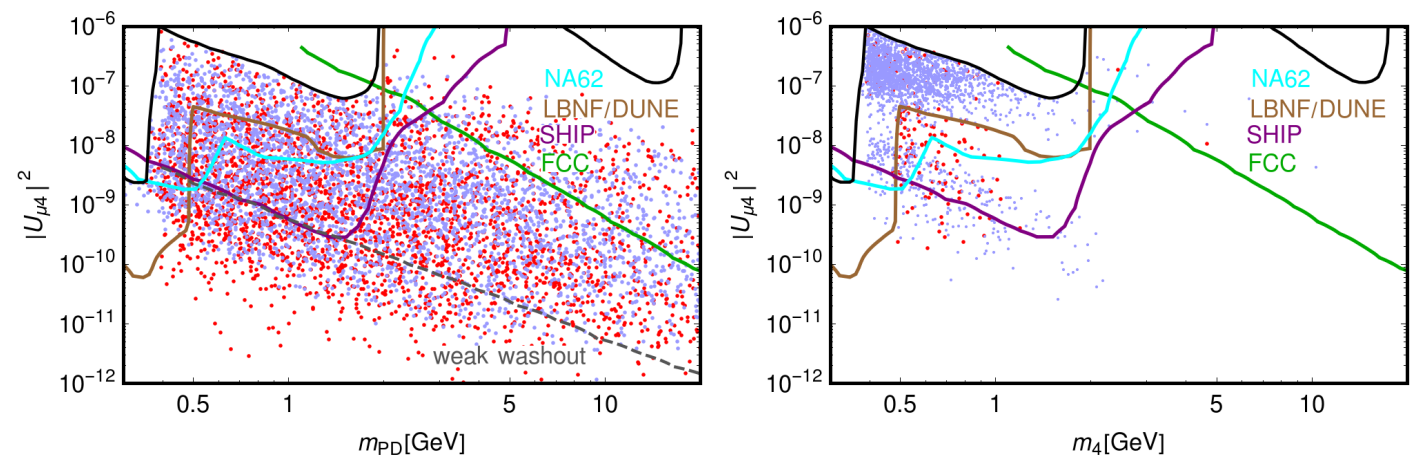

Figure 1: Mixing between active and singlet neutrino sector in the LSS-ISS (left panel) and ISS(2,2) (right-panel) for viable leptogenesis solutions. $U_{\mu 4}$ represents the mixing of these neutrinos with the muonic flavour. The region above the black line is excluded by the combination of existing bounds while the coloured lines refer to the sensitivity curves of the planned future experiments NA62, LBNF/DUNE, FCC-ee and SHiP. Solutions corresponding to the normal (inverted) hierarchy are shown in blue (red).

The viable (i.e. with $Y_{B}$ compatible with the present measurements) solutions are shown in fig. 1 in the plane $\left(m_{\mathrm{PD}, 4}, U_{\mu 4}\right)$, where $m_{\mathrm{PD}}$ and $m_{4}$ are the mass scales of the neutrinos responsible of the leptogenesis in the LSS-ISS and ISS $(2,2)$ models respectively. Notice that in the case of the ISS model we assume that only the lightest pseudo-dirac neutrino is involved in ARS oscillations. As evident from Fig. 1 successful leptogenesis is ensured over a large portion of parameter space in the LSS-ISS model while it is slightly more troublesome for the ISS $(2,2)$. In this last case the size of the Yukawa couplings increases with the masses of the neutrinos. For $m_{4} \gtrsim 3 \mathrm{GeV}$ the heavy neutrinos get close or even into thermal equilibrium causing a too strong wash-out of the baryon asymmetry. For both models the parameter space corresponding to viable leptogenesis lies within the sensitivity of current experiments, as NA62, as well as proposed future experiments as SHiP, DUNE and FCC-ee. Despite giving successful leptogenesis, the ISS $(2,3)$ resulted, however, ruled out since viable solutions were characterized by too high mixing angles between the DM and active leptons, causing its overproduction in the Early Universe.

\section{Conclusions}

The Linear and Inverse See-Saw realize the ARS leptogenesis in a technically natural way since the small mass-splitting between the heavy neutrinos is due to a tiny violation of the lepton number. We have shown, through a thorough numerical study, that viable leptogenesis can be obtained in region of parameters space which can be tested at present and future facilities.

Acknowledgements: G.A. warmly thanks the organizers of the NOW conference for this great event.

\section{References}

[1] E. K. Akhmedov, V. A. Rubakov and A. Y. Smirnov, Phys. Rev. Lett. 81 (1998) 1359 doi:10.1103/PhysRevLett.81.1359 [hep-ph/9803255].

[2] A. Abada and M. Lucente, Nucl. Phys. B 885 (2014) 651 doi:10.1016/j.nuclphysb.2014.06.003 [arXiv:1401.1507 [hep-ph]]. 
[3] A. Abada, G. Arcadi and M. Lucente, JCAP 1410 (2014) 001 doi:10.1088/1475-7516/2014/10/001 [arXiv:1406.6556 [hep-ph]].

[4] T. Asaka, S. Eijima and H. Ishida, JCAP 1202 (2012) 021 doi:10.1088/1475-7516/2012/02/021 [arXiv:1112.5565 [hep-ph]].

[5] A. Abada, G. Arcadi, V. Domcke and M. Lucente, JCAP 1712 (2017) no.12, 024 doi:10.1088/1475-7516/2017/12/024 [arXiv:1709.00415 [hep-ph]]. 